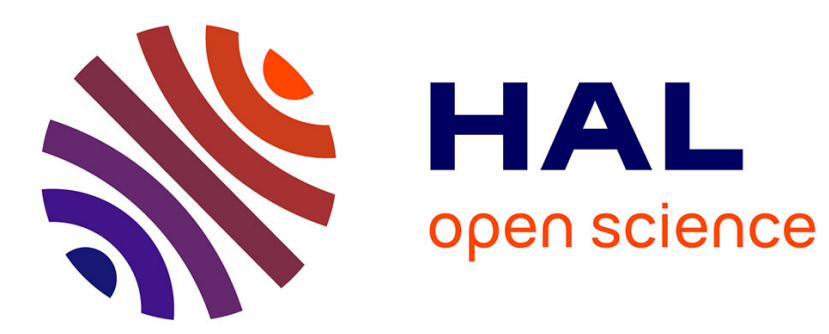

\title{
Defining and measuring paradoxical (REM) sleep in animal models of sleep disorders
}

Christelle Peyron, Sébastien Arthaud, Manon Villalba, Patrice Fort

\section{To cite this version:}

Christelle Peyron, Sébastien Arthaud, Manon Villalba, Patrice Fort. Defining and measuring paradoxical (REM) sleep in animal models of sleep disorders. Current Opinion in Physiology, 2020, 15, pp.203-209. 10.1016/j.cophys.2020.03.008 . hal-02996296

\section{HAL Id: hal-02996296 \\ https://hal.science/hal-02996296}

Submitted on 2 Dec 2020

HAL is a multi-disciplinary open access archive for the deposit and dissemination of scientific research documents, whether they are published or not. The documents may come from teaching and research institutions in France or abroad, or from public or private research centers.
L'archive ouverte pluridisciplinaire HAL, est destinée au dépôt et à la diffusion de documents scientifiques de niveau recherche, publiés ou non, émanant des établissements d'enseignement et de recherche français ou étrangers, des laboratoires publics ou privés. 
Defining and measuring paradoxical (REM) sleep in animal models of sleep disorders

$$
\text { Peyron Christelle }{ }^{1,2} \text {, Arthaud Sébastien }{ }^{1} \text {, Villalba Manon }{ }^{1} \text {, Fort Patrice }{ }^{1}
$$

${ }^{1}$ Center for Research in Neuroscience of LYON (CRNL), SLEEP team, CNRS UMR 5292, INSERM U1028, University of Lyon, NeuroCampus Michel Jouvet, Centre Hospitalier Le Vinatier, 95 Boulevard Pinel, 69675 Bron cedex, France.

${ }^{2}$ Corresponding author: Peyron Christelle, peyron@sommeil.univ-lyon1.fr 


\section{Highlights:}

* A consensual definition for transitions from NREM into REM sleep is needed to compare data obtained in different laboratories;

* The use of multi-parametric polysomnography is of great help to score vigilance states in animal models of sleep disorders;

* Video-recording synchronized to polysomnography is necessary to analyze vigilance states in animal models of sleep pathologies in order to appropriately recognize REM sleep and differentiate it from potential dissociated states. 


\section{ABSTRACT:}

In mammals including humans, the convention defining rapid-eye-movement (REM) sleep also called paradoxical sleep - states that it is mainly characterized by a low voltage fast electrocortical rhythms, rapid eye movements and a muscle atonia occasionally accompanied by brief distal twitches. When facing sleep pathologies in humans or experimentally-induced dissociated states in animals, all these criteria are not always met and identifying REM sleep becomes thus a challenge. Here we review such criteria and report the adjustments proposed to identify and measure REM sleep in animal models of sleep pathologies such as REM sleep Behavior Disorder and narcolepsy type 1 . We also argue that video-recordings synchronized to multi-parametric polysomnography are mandatory to recognize REM sleep and differentiate it from dissociated states in animal models of sleep disorders. 


\section{INTRODUCTION}

In mammals including humans, the convention defining rapid-eye-movement (REM) sleep also called paradoxical sleep - states that it is characterized by a low voltage fast electrocortical rhythms highly enriched in theta frequency (coined as cortical activation), with loss of awareness and consciousness marked by reduced responsiveness to external stimulations, rapid eye movements although eye-lids are closed, body paralysis due to an atonia of skeletal muscles occasionally accompanied by brief distal twitches and whiskers/face movements, irregular heart and respiratory rates and cessation of internal heat production. Vigilance states are well organized into ultradian cycles with the succession of wakefulness (W) to NREM sleep to REM sleep. REM sleep is always preceded by NREM sleep and it ends in its great majority by $\mathrm{W}$ or at least a micro-arousal. REM sleep is consolidated in 4 to 5 episodes during the night in humans but its daily duration and distribution vary greatly from species to species likely due to evolutionary and ecological constraints.

The above canonical definition of REM sleep [1] is primarily based on behavioral and physiological observations made on humans and a great number of mammals including cats, monkeys, dogs, rats, and more recently wild-type and transgenic mice (ref. Libourel sur REM sleep par ex). As animals used to model sleep disorders are generally mammalians, the above definition should apply to our studies. However, when facing pathologies or experimentally-induced dissociated states in animals, all these pathognomonic criteria are not always met and identifying REM sleep becomes a challenge. Here we review the criteria used to define REM sleep and report the adjustments proposed to identify and measure 
REM sleep in animal models of sleep disorders such as REM sleep Behavior Disorder (RBD) and narcolepsy type 1.

\section{MEASURING REM SLEEP IN PHYSIOLOGICAL CONDITIONS}

Polysomnography (PSG) is the gold standard for assessing REM sleep due to the high accuracy of this method for identifying vigilances states. It uses electroencephalography (EEG) - and/or local field potentials (LFP) - to measure cortical activity and hippocampal rhythmic activities (including theta oscillations) and neck electromyography (EMG) to visualize muscle atonia, which is sufficient in physiological conditions (Figure 1). It is routinely associated in humans with electrooculography (EOG) to capture eye-movements and recording of respiratory and cardiac rhythms and blood oxygen saturation but these additional physiological parameters are rarely measured in animals. It is indeed quite common nowadays to use only one lead for EEG and one for EMG in mouse PSG, mainly due to a limited space for electrodes.

Standard sleep scoring methods in rodents take in consideration high activity in nuchal EMG or animal movements from video recordings to differentiate active $W$ from sleep $[2,3]$. Recently it was also proposed to use olfactory bulb gamma power instead of EMG for on-line analysis to avoid confusion with fear immobile behaviors [4]. However, differentiation between NREM sleep and REM sleep necessitates measures of selected frequency bands amplitude from the parietal (sometimes with frontal) EEG - mainly delta ( $\delta: 0-4 \mathrm{~Hz})$ and theta $(\theta: 4-8 \mathrm{~Hz})$. Most of algorithms developed so far to score vigilance states automatically are based on such analysis [2-4]. 
Sleep is classically scored from $30 \mathrm{sec}$ epochs in humans and cats; 4-10 sec epochs are however more appropriated in rodents since sleep is more fragmented (5 to $120 \mathrm{sec}$ duration of REM sleep bouts in mice for example, [5]).

\section{EEG features during REM sleep}

In baseline condition, REM sleep is characterized by a predominant hippocampal $\theta$ rhythm that is accompanied by a cortical activation with high frequency band, also called fast gamma $(\gamma)$, activity [6]. Both, $\theta$ and $\gamma$ are easily measured with an EEG electrode placed above the parietal cortex (or LFP in intra-hippocampus) and a neutral electrode above the cerebellum. Theta rhythm generation requires an intact medial septum [7]. The optogenetic inactivation of the GABAergic neurons within the medial septum reduces power by $65 \%$ without altering other EEG frequency bands indicating that these neurons play a major role in the expression of $\theta$ oscillations [8].

Hippocampal and cortical phase-amplitude $\theta-\gamma$ coupling is prevalent during REM sleep $[9,10]$. Further analyses distinguished peaks within fast oscillations (middle $\gamma: 50-90 \mathrm{~Hz}$ and high $\gamma$ : $110-160 \mathrm{~Hz})$ in addition to the predominant $\theta$ rhythm $(6-10 \mathrm{~Hz})$ [9]. Interestingly these authors also showed that middle $\gamma$ power $(50-90 \mathrm{~Hz})$ increases during the REM sleep bouts as well as coupling values between $\theta$ and middle $\gamma$.

\section{Transitions into REM sleep}

One of the difficulties in defining REM sleep, that found no clear consensus, stands in transition from NREM sleep to REM sleep (Figure 2). When to declare that the state of REM 
sleep is established? Is the transition NREM to REM sleep itself a specific, so called intermediate state (that should be associated with specific functions) ? Should it be considered as REM sleep or NREM sleep or scored separately? Sanchez-Lopez et al [11] have recently analyzed EEG, EOG and EMG signals during transition periods and found that they follow a well-defined sequence with cortical changes ( $\delta$ waves extinction and $\theta$ plus $\gamma$ waves occurrence) preceding those affecting the motor system. These authors defined the end of the transition period by the complete loss of muscle tone that coincides with the maximum amplitude of the $\gamma$ oscillation. Duran et al [12] showed that depending on the placement of the electrodes (frontal vs hippocampal) REM sleep onset would be considered differently. In our laboratory, we score as REM sleep when $\delta$ power is minimum, $\theta$ power maximal on both frontal and parietal EEG while muscle tone is abolished (Figure 2). The absence of consensus on that matter may introduce not only differences in data analyses from lab to lab but also biases in the interpretation of data although transition periods are quite brief ( $2 \%$ of total time in rats [12]) in physiological condition.

\section{REM sleep homeostatic regulation}

A large body of controversial literature has been produced trying to reveal the nature of REM sleep homeostatic regulation, the most recent ones suggesting a unique mode of regulation. Indeed, unlike NREM sleep that is compensated by increases in depth (i.e. increase in slow wave activity power) and quantities, REM sleep is only recovered by increasing its amount, $\theta$ power remaining unchanged [5]. We further found that the need to recover from REM sleep deficit is dealt with by favoring transitions from NREM to REM sleep and maintaining REM sleep. The first strategy used to compensate for REM sleep deficit is to 
increase the mean duration of REM sleep bouts without changing their occurrence; but when insufficient, multiplying the entrance into REM sleep may also be favored. This strategy is done at the expense of NREM sleep and W, both having less opportunity to occur but once entered into, both states appear stabilized and consolidated, as well.

A large body of literature has demonstrated that NREM sleep pressure increases during the time spent awake as illustrated in the process $S$ model [13]. A recent elegant study further showed that sleep homeostasis is strongly driven by a sub-state of $W$ that is $\theta$-dominated [14]. We recently found however, that unlike NREM sleep, REM sleep recovery after REM sleep specific deprivation is positively correlated to the amount of REM sleep lost but not to the amount of time spent from the last REM sleep episode [5].

These above observations stand in the context of acute sleep deprivation paradigms. However, when total sleep is chronically restricted, REM sleep settles after the first day of sleep restriction on a persistent elevated level. Its total duration does not progressively increase over the days - although it increases as a percentage of total sleep time - while both REM sleep and NREM sleep debts keep accumulating (Kim et al, 2017).

\section{MEASURING REM SLEEP IN ANIMAL MODELS OF SLEEP DISORDERS}

As mentioned in the introduction, the association of two major parameters defines REM sleep in rodents: muscle atonia (nuchal EMG) and a cortical EEG enriched in theta frequency. The dysregulation of one of these two REM sleep-defining criteria in the context of sleep pathologies results in major difficulties to properly score PSG recordings and assign a vigilance state with minimized doubt.

\section{REM sleep Behavior Disorder in rats and mice}


In REM sleep Behavior Disorder (RBD, [15]) - a REM sleep specific parasomnia - and its preclinical models only available in rodents to date $[16,17]$, REM sleep is marked by the loss of muscle atonia facilitating the occurrence of abnormal, uncontrolled motor activities (though not always detectable on nuchal EMG), while EEG rhythmic activities (mainly theta oscillations) remain unmodified compared to natural REM sleep, a dissociated vigilance state difficult to differentiate from active W using PSG only.

In that context, some advices may be given to minimize errors and misinterpretations in the scoring of REM sleep/RBD bouts. First of all, natural REM sleep episodes or pathologic RBDs only emerge from NREM and not from inappropriate direct transition from wake as seen in narcolepsy (figure 3). Moreover, daily REM sleep and wake amounts are not significantly modified in RBD patients and animals [15-17], indicating that in both cases the ultradian rhythm of REM sleep is unaffected. Besides, as routinely done in clinical practice, our recent studies in rodents illustrate the clear benefit of complementary video acquisition timely synchronized to PSG for a correct discrimination of REM sleep/RBDs from active wake $[16,17]$. Using video recording, we were able to check whether eyelids were closed as expected during REM sleep and RBDs (while likely open during wakefulness) and to scrutinize the REM sleep-specific vibrissae movements. We were also able to analyze the collapsed posture associated with abnormal movements of body parts shaking in random sequences, that were clearly visually different from natural postures of coordinated locomotion coherent with animal's motor behaviors during W. One additional signal that may be relevant to record is the EOG since REMs are known to be a common cardinal parameter of REM sleep and RBDs. They are qualitatively different from those during $W$ reflecting gaze movements. Finally, EMG recordings of other body territories in addition to neck muscles (i.e. distal extremities, facial or masseter muscles) may also help to 
discriminate natural twitches from abnormal movements during REM sleep and RBD, respectively.

\section{Narcolepsy type 1 in animal models}

The first animal model of narcolepsy was developed in dogs. A colony of Doberman pinchers and Labrador retrievers was bred, both carrying autosomal recessive mutations of the hypocretin receptor 2 (Lin et al, 1999), mutations never reported in human narcolepsy to date. Although the cause of canine narcolepsy is clearly different to that in patients, the hypocretin/orexin system is impaired in both cases. The canine model still allowed for many physiology and pharmacology observations that provided a better understanding of the disease (Nishino and Mignot, 1997; Tonokura et al, 2007). REM sleep in narcoleptic dogs showed same characteristics as in controls, the total amount of time spent in wake and sleep during the daytime was not altered but, their wake and sleep patterns were fragmented (Nishino et al, 2000). Canine cataplexy events presented a bilateral muscle atonia along with a REM sleep-like EEG with hippocampal theta activity (Kushida 1985) rendering difficult to the differentiate cataplexy from SOREM ??? if only PSG is considered. The behavioral context has been very helpful to distinguish cataplexy from REM sleep. In dogs, the loss of muscle tone starts in the hind legs and progresses to the front legs and the neck and, cataplexy is strongly triggered by food and play (Fujiki 2002).

The canine model of narcolepsy is rarely used nowadays at the benefit of murine models, easier to handle and mimicking similar human impairments. 
Several murine models of narcolepsy are available, constitutive or conditional, genetic or neuroinflammatory, with progressive or rapid onset, all targeting the hypcretin neuropeptides or the hypocretin neurons. All show a REM sleep PSG similar to their wildtype counterparts [18-21]. Power spectrum EEG analyses during REM sleep show undistinguishable profiles in narcoleptic and wild-type mice [22]. However, confusion can be made between sleep onset into REM sleep from active $W$ and one of the main symptoms of narcolepsy disease, cataplexy.

Cataplexy is a sudden loss of muscle tone during active $\mathrm{W}$ triggered by strong positive emotion. An international consensual definition of cataplexy was proposed by a large group of experts [23]: Cataplexy in mice should last for at least $10 \mathrm{sec}$ as characterized by a muscle atonia, be preceded by at least $40 \mathrm{sec}$ of active $\mathrm{W}$ and terminate by a rapid behavioral recovery to active W. During cataplexy, muscle tone should be absent and the EEG dominated by theta oscillations.

Mouse cataplexy shares many features with REM sleep. When a mouse falls from active wake into cataplexy, EEG changes from a typical W pattern to one very similar to REM sleep (Figure 4). Once cataplexy is fully established, EEG is dominated by high amplitude theta activity with an EEG power spectrum very similar to REM sleep. Sometimes, sustained mouse cataplexy seems to evolve into REM sleep as has been reported for some episodes in patients. Several attempts have been made to identify biomarkers of cataplexy in mice. Bursts of hyper-synchronous activity were found to be frequent when recording EEG frontal derivations [24] although a similar feature has been also described during REM sleep at lower density or in mice knocked-out for histidine decarboxylase, the enzyme synthesizing histamine $[25,26]$. Others have used masseter muscular atonia to confirm cataplexy [27] or 
" abrupt » versus " gradual » entrance into arrests to differentiate cataplexy from SOREM [28]. Although not easy to decipher at first, the behavioral context and evolution tremendously helps to distinguish cataplexy from REM sleep or other behavioral attacks: the way the animal collapses during cataplexy is very informative. Video recording in association with PSG is thus necessary to accurately distinguish cataplexy from onset into REM sleep or other attacks.

As antidepressants are used to treat cataplexy, a few studies have tested their effect on narcoleptic mice and dogs. Mostly they found a reduction of both cataplexy and REM sleep (Willie et al, 2003; Burgess et al, 2010; Schmidt et al, 2016). Some drugs however, modulating D2-receptor activity, did modulate the amount of cataplexy without affecting REM sleep (Burgess et al, 2010; Okura et al 2000).

\section{CONCLUSION}

Based on observations of pathologies, one could wonder what are the essential criteria defining REM sleep. If muscle atonia were a pre-requisite to define the state of REM sleep, one would say that REM sleep in RBD is not REM sleep. However, if theta-gamma coupling on the parietal EEG was sufficient to define the state, one could declare that cataplexy is REM sleep. We would argue that it is essential to use multi-parameters (including several EEG, EOG and several EMG signals with heart beat and body temperature) and most importantly video-recording synchronized to PSG when analyzing sleep in animal models of pathologies to appropriately recognize REM sleep and differentiate it from dissociated states. 


\section{Financial support}

CP and PF are supported by CNRS, SA by the University Lyon 1 and MV doctoral fellowship is granted by Bioprojet.

Conflict of interest statement:

None to declare 


\section{REFERENCES}

1. Jouvet M: [The paradoxical phase of sleep]. Int J Neurol 1965, 5:131-150.

2. Libourel PA, Corneyllie A, Luppi PH, Chouvet G, Gervasoni D: Unsupervised online classifier in sleep scoring for sleep deprivation studies. Sleep 2015, 38:815-828.

3. Kassiri H, Chemparathy A, Salam MT, Boyce R, Adamantidis A, Genov R: Electronic Sleep Stage Classifiers: A Survey and VLSI Design Methodology. IEEE Trans Biomed Circuits Syst 2017, 11:177-188.

4. Bagur S, Lacroix MM, de Lavilleon G, Lefort JM, Geoffroy H, Benchenane K: Harnessing olfactory bulb oscillations to perform fully brain-based sleep-scoring and real-time monitoring of anaesthesia depth. PLOS Biol 2018, 16:e2005458.

5. Arthaud S, Libourel PA, Luppi PH, Peyron C: Insights into paradoxical (REM) sleep homeostatic regulation in mice using an innovative automated sleep deprivation method. Sleep 2020.

6. Maloney KJ, Cape EG, Gotman J, Jones BE: High-frequency gamma electroencephalogram activity in association with sleep-wake states and spontaneous behaviors in the rat. Neuroscience 1997, 76:541-555.

7. Mitchell SJ, Rawlins JN, Steward O, Olton DS: Medial septal area lesions disrupt theta rhythm and cholinergic staining in medial entorhinal cortex and produce impaired radial arm maze behavior in rats. J Neurosci 1982, 2:292-302.

8. Boyce R, Glasgow SD, Williams S, Adamantidis A: Causal evidence for the role of REM sleep theta rhythm in contextual memory consolidation. Science 2016, 352:812-816.

9. Bandarabadi M, Boyce R, Gutierrez Herrera C, Bassetti CL, Williams S, Schindler K, Adamantidis A: Dynamic modulation of theta-gamma coupling during rapid eye movement sleep. Sleep 2019, 42. 
10. Cavelli M, Rojas-Libano D, Schwarzkopf N, Castro-Zaballa S, Gonzalez J, Mondino A, Santana N, Benedetto L, Falconi A, Torterolo P: Power and coherence of cortical high-frequency oscillations during wakefulness and sleep. Eur J Neurosci 2018, 48:2728-2737.

11. Sanchez-Lopez A, Silva-Perez M, Escudero M: Temporal dynamics of the transition period between nonrapid eye movement and rapid eye movement sleep in the rat. Sleep 2018, 41.

* The transition from NREM to REM is a progressive process. No consensus exist to this date to when to declare REM sleep as fully established. In this study the authors consider the state of REM sleep when high frequency oscillations have reached their maximum amplitude.

12. Duran E, Oyanedel CN, Niethard N, Inostroza M, Born J: Sleep stage dynamics in neocortex and hippocampus. Sleep 2018, 41.

* This study illustrates the spatial dynamic of theta rhythms occurrence, highlighting the importance of electrodes placement for the identification of vigilance states.

13. Borbely AA: A two process model of sleep regulation. Hum Neurobiol 1982, 1:195-204.

14. Vassalli A, Franken P: Hypocretin (orexin) is critical in sustaining theta/gamma-rich waking behaviors that drive sleep need. Proc Natl Acad Sci U S A 2017, 114:E5464E5473.

* This study brings to new insights of the homeostatic regulation of NREM sleep showing that need for NREM sleep is in fact driven by a sub-state of wakefulness, highly rich in theta/gamma activity.

15. Dauvilliers Y, Schenck CH, Postuma RB, Iranzo A, Luppi PH, Plazzi G, Montplaisir J, Boeve B: REM sleep behaviour disorder. Nat Rev Dis Primers 2018, 4:19. 
16. Valencia Garcia S, Brischoux F, Clement O, Libourel PA, Arthaud S, Lazarus M, Luppi PH, Fort P: Ventromedial medulla inhibitory neuron inactivation induces REM sleep without atonia and REM sleep behavior disorder. Nat Commun 2018, 9:504.

* This study demonstrates that the GABAergic/glycinergic neurons of the ventromedial medulla are necessary to maintain muscle inhibition during REM sleep. With this study, the authors created a rat model of RBD recapitulating most of the symptoms of this human disease and showed that video-recordings are mandatory to analyze atonia impairment and identify REM sleep accurately.

17. Valencia Garcia S, Libourel PA, Lazarus M, Grassi D, Luppi PH, Fort P: Genetic inactivation of glutamate neurons in the rat sublaterodorsal tegmental nucleus recapitulates REM sleep behaviour disorder. Brain 2017.

18. Chemelli RM, Willie JT, Sinton CM, Elmquist JK, Scammell T, Lee C, Richardson JA, Williams SC, Xiong $Y$, Kisanuki $Y$, et al.: Narcolepsy in orexin knockout mice: molecular genetics of sleep regulation. Cell 1999, 98:437-451.

19. Hara J, Beuckmann CT, Nambu T, Willie JT, Chemelli RM, Sinton CM, Sugiyama F, Yagami K, Goto K, Yanagisawa M, et al.: Genetic ablation of orexin neurons in mice results in narcolepsy, hypophagia, and obesity. Neuron 2001, 30:345-354.

20. Tabuchi S, Tsunematsu T, Black SW, Tominaga M, Maruyama M, Takagi K, Minokoshi Y, Sakurai T, Kilduff TS, Yamanaka A: Conditional ablation of orexin/hypocretin neurons: a new mouse model for the study of narcolepsy and orexin system function. J Neurosci 2014, 34:6495-6509.

21. Bernard-Valnet R, Yshii L, Queriault C, Nguyen XH, Arthaud S, Rodrigues M, Canivet A, Morel AL, Matthys A, Bauer J, et al.: CD8 T cell-mediated killing of orexinergic 
neurons induces a narcolepsy-like phenotype in mice. Proc Natl Acad Sci U S A 2016, 113:10956-10961.

22. Roman A, Meftah S, Arthaud S, Luppi PH, Peyron C: The inappropriate occurrence of rapid eye movement sleep in narcolepsy is not due to a defect in homeostatic regulation of rapid eye movement sleep. Sleep 2018, 41.

23. Scammell TE, Willie JT, Guilleminault C, Siegel JM: A consensus definition of cataplexy in mouse models of narcolepsy. Sleep 2009, 32:111-116.

24. Vassalli A, Dellepiane JM, Emmenegger Y, Jimenez S, Vandi S, Plazzi G, Franken P, Tafti M: Electroencephalogram paroxysmal theta characterizes cataplexy in mice and children. Brain 2013, 136:1592-1608.

25. Bastianini S, Silvani A, Berteotti C, Lo Martire V, Zoccoli G: High-amplitude theta wave bursts during REM sleep and cataplexy in hypocretin-deficient narcoleptic mice. J Sleep Res 2012, 21:185-188.

26. Bastianini S, Lo Martire V, Berteotti C, Silvani A, Ohtsu H, Lin JS, Zoccoli G: Highamplitude theta wave bursts characterizing narcoleptic mice and patients are also produced by histamine deficiency in mice. J Sleep Res 2016, 25:591-595.

27. Snow MB, Fraigne JJ, Thibault-Messier G, Chuen VL, Thomasian A, Horner RL, Peever J: GABA Cells in the Central Nucleus of the Amygdala Promote Cataplexy. J Neurosci 2017, 37:4007-4022.

28. Willie JT, Chemelli RM, Sinton CM, Tokita S, Williams SC, Kisanuki YY, Marcus JN, Lee C, Elmquist JK, Kohlmeier KA, et al.: Distinct narcolepsy syndromes in Orexin receptor2 and Orexin null mice: molecular genetic dissection of Non-REM and REM sleep regulatory processes. Neuron 2003, 38:715-730. 


\section{LEGEND}

Figure 1: Representative examples of EMG, EOG, LFP and EEG traces during wakefulness NREM and REM sleep of a wild-type C57BI6 mouse. EEG, electroencephalograpy; EMG, electromyography; EOG, electrooculography; Fr, frontal cortex; LFP, local field potential; OB, olfactory bulb; Par, parietal cortex.

Figure 2: Representative EEG and EMG traces of a transition from NREM to REM sleep in a wild-type C57BI6 mouse. Power spectra of $5 \mathrm{sec}$ epochs are illustrated below the corresponding EEG trace of the activity of the parietal cortex. TR, transition state.

Figure 3: Representative illustrations of EEG-EMG traces in a wild-type mouse (top traces), a mouse model of REM sleep behavior disorder (middle traces) and a orexin-ko narcoleptic mouse having a cataplexy attack (bottom traces). Note that all mice are C57Bl6/J genetic background. W, wakefulness. 